\title{
Beamforming-based Interference Cancellation for Multiuser MIMO Downlink Communications
}

\author{
Yen-Hsu Chiang and Hsi-Pin Ma \\ Department of Electrical Engineering National Tsing Hua University, Hsinchu, Taiwan, R.O.C. \\ Email: yschiang@larc.ee.nthu.edu.tw, hp@ee.nthu.edu.tw
}

\begin{abstract}
In this paper, the author proposes an interference cancellation precoding method (MB-BD) for multiuser MIMO downlink systems. Maximum beamforming (MB) is used for SNR improvement but just in single-substream transmission. MB-BD is based on maximum beamforming for multi-substream transmission and can improve the received SNR at each antenna of desired users because it uses the sum space of the eigenvectors which correspond to the strong eigenvalues (eigen modes) to transmit data for desired users. Comparing to block diagonalization (BD), MB-BD has 6dB gain if we set constraint of BER at $10^{-3}$ and the sum rate of the proposed method is identical to $\mathrm{BD}$.

MB-BD has lower computational complexity than BD because it needs only to do one SVD (or EVD) and one QR decomposition for computing the precoding matrix of each user. We can get about $37.5 \%$ reduction of computational complexity at transmitter. To enhance the detection performance and lower computational complexity, we use simplified maximum likelihood (SML) at each receiver. Comparing to original ML, we can get about $33.33 \%$ reduction of computational complexity at receiver.
\end{abstract}

\section{INTRODUCTION}

Multiple-input multiple output (MIMO) communication is the key technology for achieving high data rates in bandlimited wireless communication systems. Generally speaking, a multiuser MIMO downlink system means that each user or mobile has more than one received antenna to receive the data from base station (BS). Multiuser spatial multiplexing (MUSM) system is a case of MIMO broadcast (MIMO-BC) communication problem. In this paper, spatial multiplexing multiuser MIMO downlink is investigated, one BS aim to communicate with several co-channel users in the same frequency and time slots. Precoding is required for MIMO systems for the low complexity at mobile units and large diversity gain. So channel state information (CSI) is known at transmitter for this kind "closed loop" MIMO systems. In the case, it is necessary to design a reliable transmission schemes that are able to suppress the co-channel interference (CCI) at the end users.

In [1], Spencer gave an introduction to the multi-user MIMO downlink, he described two classes of solutions to deal with the interference. The first uses various types of linear precoding or beamforming methods at BS. The second uses nonlinear dirty paper coding method [2] which is optimal theoretically to achieve the capacity region [3], [4], and [5] but difficult to implement in practical. The well-known simplest for multiuser precoding method is to pre-inverse the multiuser matrix channel by normalized zero-forcing (ZF) or minimum mean square error (MMSE) [6] for the special case of a single receive antenna per user. Block diagonalization (BD) [7] is a generalization of the zero forcing channel inversion to the case where each receiver is equipped with multiple antennas. For minimum mean-squared error of channel inversion, the regularized channel inversion (RCI) [8] for multiple antennas for each user is proposed.

The iterative precoding method has good error performance but results in high computational complexity. Nu-SVD [9] is base on the null-space criterion and T-MMSE [10] is a scheme based on the minimum total MSE criterion under a total transmit power constraint. Lee [8] shows that RCI has better sum rate performance than Nu-SVD and T-MMSE but does not require any receiver processings. In this paper, we propose a new transmitted method for mitigating the multiuser interference in multiuser downlink systems. For lower computational complexity, we focus on the non-iterative and linear methods for transmit precoding matrix.

The motivations of this paper is to design a precoding algorithm that is able to not only cancel multiuser interference but also improve the received SNR. To point out some features of the proposed system as follows:

A. Multiuser Interference Cancellation As conventional block diagonalization (BD) [7], the proposed method (MB-BD) provide an alternative interference cancellation method for multiuser MIMO downlink systems.

B. Better Diversity Gain Comparing to conventional block diagonalization (BD) [7], the proposed scheme (MBBD) has $6 \mathrm{~dB}$ gain when we set constraint of BER at $10^{-3}$.

C. Better Error Performance for Imperfect Channel Information at Transmitter We take mean square error (MSE) of channel state information (CSI) into consideration, the simulation result shows that the proposed method has better error performance when imperfect channel information at transmitter.

This paper is organized as follows: In Section II, we describe total system model. In Section III, we introduce some linear precoding methods of multiuser MIMO downlink systems. Section IV shows the proposed precoding scheme. Section $\mathrm{V}$ presents the simulation results of the proposed algorithm. Finally, Section VI makes the conclusions for this 
paper.

\section{SySTEM MODEL}

In Figure 1, we assume a BS employs $M$ transmit antennas and communicates with $K$ users simultaneously. The $j^{t h}$ user $(j=1,2, \ldots, K)$, has $n_{j}$ receive antennas and the total received antennas of all users is $N=\sum_{j=1}^{K} n_{j}$.

The channel matrix $H_{j}$ for the $j^{\text {th }}$ user is an $n_{j}$ by $M$ matrix. The $(p, q)$ entry of $H_{j}$ denotes the path gain from BS antenna $q$ to antenna $p$ for $j^{t h}$ user. The complex entries are independently and identically distribution (i.i.d.) Gaussian random variables with zero mean and unit variance.

We also define that $l_{j}$ is the number of data streams for $j^{t h}$ user. The symbol vector $x_{j}$ for $j^{t h}$ user is a $l_{j} \times 1$ vector which can be written as

$$
x_{j}=\left[x_{j, 1} x_{j, 2} \cdots x_{j, l_{j}}\right]^{T} .
$$

The linear precoding matrix $T_{j}$ for user $\mathrm{j}$ is a $M \times l_{j}$ matrix

$$
T_{j}=\left[t_{j, 1} t_{j, 2} \cdots t_{j, l_{j}}\right]
$$

The receiver data vector $y_{j}=\left[y_{j, 1} y_{j, 2} \cdots y_{j, n_{j}}\right]^{T}$ at the $j^{\text {th }}$ user can be written as

$$
y_{j}=H_{j} T_{j} x_{j}+H_{j} \sum_{i \neq j} T_{i} x_{i}+w_{j},
$$

where $w_{j}=\left[w_{j, 1} w_{j, 2} \cdots w_{j, n_{j}}\right]^{T}$ for $j=1, \ldots, K$ and $i=1, \ldots, n_{j}$. The elements of $w_{j}$ are i.i.d. Gaussian random variables with zero mean and variance $\sigma_{n}^{2}$. The network channel is

$$
H_{s}=\left[H_{1} H_{2} \ldots H_{K}\right]^{T} .
$$

The corresponding signals at all the users can be written as

$$
y_{s}=H_{s} T_{s} x_{s}+w_{s}
$$

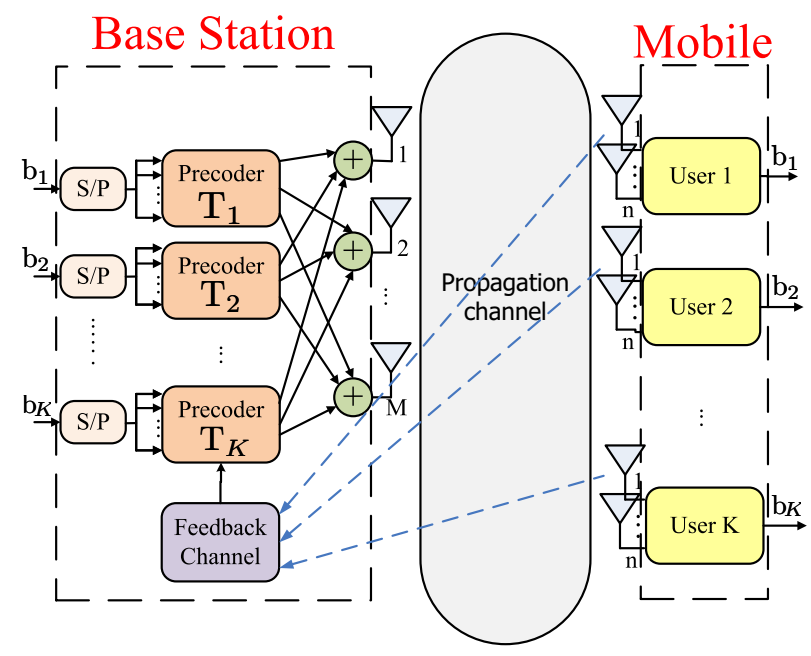

Fig. 1. The block diagram of multiuser MIMO downlink systems.

\section{LinEAR PRECOdING Methods}

\section{A. Block Diagonalization}

The advantage of block diagonalization (BD) [7] is that it can eliminate the multi-user interference (MUI) by placing all the unintended users' data at null space of the intended users' channels.

BD doesn't take consideration on the noise so the sum rate doesn't grow linearly with the number of users due to the noise enhancement.

\section{B. Regularized Channel Inversion}

Also called minimum mean-squared error channel inversion (MMSE-CI). When channel is rank-deficient, a common approach to reducing the effects of noise amplification is to regularize the inverse in the $\mathrm{ZF}$ filter [11].

\section{Optimum Beamforming}

Optimum beamforming is the maximal ratio combining (MRC) solution that provides the maximum output signal-tonoise ratio (SNR), so it is also called maximal beamforming (MB). In a MIMO channel with interference, the optimum beamforming is the minimum-mean-square-error (MMSE) solution and is also referred to as the optimum combining (OC) scheme in that it maximizes the output signal to interferenceplus-noise ratio (SINR) [12].

MB can be used in multiuser MIMO systems. Nishimoto [13] proposed an imperfect block diagonalization (IBD) method for multiuser MIMO downlink system. It has a good performance but just for single-substream transmission.

\section{THE PROPOSED PRECODING SCHEME}

We assume four transmit antennas in the base station (BS) and two users, BS transmits two data streams for each user and every user has two antennas to received data streams from BS. We assume multi-substream transmission for every user. Because the channel matrix of $i^{\text {th }}$ user is a $2 \times 4$ matrix, the eigenvalue decomposition (EVD) of $H_{i}^{H} H_{i}$ is

$$
H_{i}^{H} H_{i}=Q_{i} \Sigma Q_{i}
$$

where

$$
Q_{i}=\left[\begin{array}{llll}
q_{i, 1} & q_{i, 2} & q_{i, 3} & q_{i, 4}
\end{array}\right]
$$

Taking the eigenvectors $q_{i, 1}$ and $q_{i, 2}$ correspond to the first and second largest eigenvalue.

$W_{i}=\left[q_{1,1} q_{1,2} \ldots q_{i-1,1} q_{i-1,2} q_{i+1,1} q_{i+1,2} \ldots q_{K, 1} q_{K, 2} q_{i, 1} q_{i, 2}\right]$.

Gram-Schmidt orthonormalization (GSO) is applied to the matrix $W_{i}$, so we define $G S O\left[W_{i}\right]$ as

$$
\left[q_{1,1}^{\prime} q_{1,2}^{\prime} \ldots q_{i-1,1}^{\prime} q_{i-1,2}^{\prime} q_{i+1,1}^{\prime} q_{i+1,2}^{\prime} \ldots q_{K, 1}^{\prime} q_{K, 2}^{\prime} q_{i, 1}^{\prime} q_{i, 2}^{\prime}\right] .
$$

The precoding matrix for $i^{t h}$ user $T_{i}$ can be written as

$$
T_{i}=\left[t_{i, 1} t_{i, 2}\right]=\left[q_{i, 1}^{\prime} q_{i, 2}^{\prime}\right] .
$$


The singular value decomposition of the channel of $i^{t h}$ user, $H_{i}$, is a two by four matrix.

$$
H_{i}=\left[s_{i, 1} s_{i, 2}\right] \Sigma_{i}\left[d_{i, 1} d_{i, 2} d_{i, 3} d_{i, 4}\right]^{H}
$$

and the precoing matrix for $k^{t h}$ user $(k \neq i)$

$$
\begin{aligned}
& T_{k}=\left[\sum_{l=3}^{4} \alpha_{k, l} d_{i, l} \sum_{l=3}^{4} \beta_{k, l} d_{i, l}\right] . \\
& H_{i} T_{k}=\left[\begin{array}{ll}
s_{i, 1} & s_{i, 2}
\end{array}\right]\left[\begin{array}{cccc}
\sqrt{\lambda_{i, 1}} & 0 & 0 & 0 \\
0 & \sqrt{\lambda_{i, 2}} & 0 & 0
\end{array}\right]\left[\begin{array}{c}
d_{i, l}^{H} \\
d_{i, 2}^{H} \\
d_{i, 3}^{H} \\
d_{i, 4}^{H}
\end{array}\right] T_{k} \\
& =\left[s_{i, 1} s_{i, 2}\right]\left[\begin{array}{cccc}
\sqrt{\lambda_{i, 1}} & 0 & 0 & 0 \\
0 & \sqrt{\lambda_{i, 2}} & 0 & 0
\end{array}\right]\left[\begin{array}{c}
d_{i, l}^{H} \\
d_{i, 2}^{H} \\
d_{i, 3}^{H} \\
d_{i, 4}^{H}
\end{array}\right] \\
& \cdot\left[\sum_{l=3}^{4} \alpha_{k, l} d_{i, l} \sum_{l=3}^{4} \beta_{k, l} d_{i, l}\right] \\
& =\left[s_{i, 1} s_{i, 2}\right]\left[\begin{array}{cccc}
\sqrt{\lambda_{i, 1}} & 0 & 0 & 0 \\
0 & \sqrt{\lambda_{i, 2}} & 0 & 0
\end{array}\right]\left[\begin{array}{cc}
0 & 0 \\
0 & 0 \\
\alpha_{k, 3} & \beta_{k, 3} \\
\alpha_{k, 4} & \beta_{k, 4}
\end{array}\right] \\
& =\left[\begin{array}{ll}
0 & 0 \\
0 & 0
\end{array}\right] \text { (No multiuser interference). }
\end{aligned}
$$

$$
C_{\mathrm{MB}-\mathrm{BD}}=\sum_{i=1}^{K}\left(1+\operatorname{SINR}_{i}\right) .
$$

We assume receiver knows the channel state information and precoding matrix, so we use maximum likelihood detection (MLD) to detect the data.

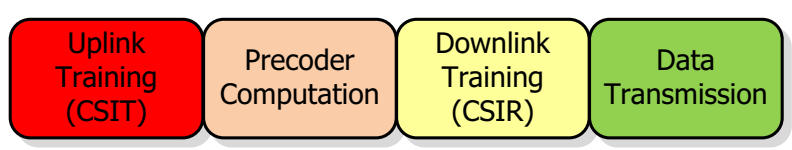

Fig. 2. Four phase multiuser MIMO protocol for forward link transmission over one coherence interval.

In [14], Gomadam proposed a two-way training methods for multi-user MIMO systems. There are four phases in the protocol for forward-link transmission over one coherence interval. We can see the Figure 2.

phase 1 Base station obtains estimates of all downlink channels ( By uplink training or downlink channel training and feedback ).

The effective channel correspond to $i^{t h}$ user can be written as

$$
\begin{aligned}
& H_{i} T_{i}=\left[s_{i, 1} s_{i, 2}\right]\left[\begin{array}{cccc}
\sqrt{\lambda_{i, 1}} & 0 & 0 & 0 \\
0 & \sqrt{\lambda_{i, 2}} & 0 & 0
\end{array}\right]\left[\begin{array}{c}
d_{i, l}^{H} \\
d_{i, 2}^{H} \\
d_{i, 3}^{H} \\
d_{i, 4}^{H}
\end{array}\right] T_{i} \\
& =\left[s_{i, 1} s_{i, 2}\right]\left[\begin{array}{cccc}
\sqrt{\lambda_{i, 1}} & 0 & 0 & 0 \\
0 & \sqrt{\lambda_{i, 2}} & 0 & 0
\end{array}\right]\left[\begin{array}{c}
d_{i, l}^{H} \\
d_{i, 2}^{H} \\
d_{i, 3}^{H} \\
d_{i, 4}^{H}
\end{array}\right] \\
& \cdot\left[\sum_{l=1}^{2} \alpha_{i, l} d_{i, l} \sum_{l=1}^{2} \beta_{i, l} d_{i, l}\right] \\
& =\left[s_{i, 1} s_{i, 2}\right]\left[\begin{array}{cccc}
\sqrt{\lambda_{i, 1}} & 0 & 0 & 0 \\
0 & \sqrt{\lambda_{i, 2}} & 0 & 0
\end{array}\right]\left[\begin{array}{cc}
\alpha_{i, 1} & \beta_{i, 1} \\
\alpha_{i, 2} & \beta_{i, 2} \\
0 & 0 \\
0 & 0
\end{array}\right] \\
& =\left[s_{i, 1} s_{i, 2}\right]\left[\begin{array}{cc}
\sqrt{\lambda_{i, 1}} \alpha_{i, 1} & \sqrt{\lambda_{i, 1}} \beta_{i, 1} \\
\sqrt{\lambda_{i, 2}} \alpha_{i, 2} & \sqrt{\lambda_{i, 2}} \beta_{i, 2}
\end{array}\right]
\end{aligned}
$$

The result shows that we use sum space of the eigenvectors which correspond to the strong eigenvalues (eigen modes) to transmit data for desired users.

We can obtain the received data vector $y_{i}$ for $i^{\text {th }}$ user

$$
\begin{aligned}
y_{i} & =H_{i} T_{i} x_{i}+H_{i} T_{k} x_{k}+w_{i} \\
& =H_{i} T_{i} x_{i}+w_{i}(k \neq i) .
\end{aligned}
$$

phase 2 MU-MIMO precoder computation at BS ( Precoder is known only at transmitter ).

phase 3 Base station transmits pilots via MU-MIMO precoding strategy of phase 2 to estimate the effective channel ( downlink effective channel training ).

phase 4 Downlink data transmission and detection.

\section{A. Complexity of the Proposed Scheme}

The complexity of SVD is $O\left(\max \left(M^{2} q, M q^{2}, q^{3}\right)\right)$ [15] at $\mathrm{BS}$, where $M$ is antenna number in the $\mathrm{BS}, q=\sum_{j=1, j \neq k}^{K} n_{j}\left(n_{j}\right.$ is $j^{\text {th }}$ user's antenna number and $K$ denotes the user number). Because we assume $M \geq \max _{k} \sum_{j=1, j \neq k}^{K} n_{j}$, the computational complexity turns out to be $O\left(M^{2} \max _{k} \sum_{j=1, j \neq k}^{K} n_{j}\right)$. The complexity is $O\left(M^{2}(K-1) n\right)$ if $n=n_{j}$, for $j=1 \ldots K$.

$\mathrm{BD}$ needs to do SVD two times, so the computational complexity of $\mathrm{BD}$ is $2 O\left(K M^{2}(K-1) n\right)$. MB-BD needs SVD (or EVD) and QR decomposition, the complexity of QR decomposition is lower than $O\left(K n^{3}\right)$ by a factor of 1.31.4 [16], MB-BD has lower computational complexity than the SVD-based approach. In addition, [17] and [15] point that QR-based method is generally much more accurate and stable numerically. The complexity of the proposed method is lower than $O\left(K M^{2}(K-1) n\right)+O\left(K n^{3}\right)=O\left(K n\left(M^{2}(K-1)+\right.\right.$ $\left.\left.n^{2}\right)\right)$. In our proposed system specifications, we set $K=2$, $n=2$, and $M=4$. We can find out that the order in BD is 
TABLE I

SYSTEM COMPLEXITY COMPARISON FOR THE PROPOSED SCHEME AND THE OTHER SYSTEMS

\begin{tabular}{|c|l|l|}
\hline \hline & $\begin{array}{l}\text { Transmitter } \\
\text { Complexity }\end{array}$ & Receiver Complexity \\
\hline \hline BD & $\begin{array}{l}2 O\left(K M^{2}(K-1) n\right) \\
{[15]}\end{array}$ & $O(n)[15]$ \\
\hline MB-BD-RxML & $<O\left(K n\left(M^{2}(K-\right.\right.$ & $O\left(C^{n} n(n+1)\right) \quad[18]$ \\
& $\left.\left.1)+n^{2}\right)\right)[16]$ & \\
\hline MB-BD-RxSML & $<O\left(K n\left(M^{2}(K-\right.\right.$ & $O(16 C) \quad[18]$ \\
& $\left.\left.\left.1)+n^{2}\right)\right)\right)[16]$ & \\
\hline
\end{tabular}

about 128 , but just 80 of the proposed method. Comparing to $\mathrm{BD}$, we can get about $\frac{128-80}{128} \times 100 \%=37.5 \%$ reduction of computational complexity at transmitter.

However, complexity is huge if we use ML detection at each mobile. In [19] and [18], the complexity is in terms of the number of complex multiplications for simplicity. For original ML detection, the complexity is $C^{N_{t x}} N_{r x}\left(N_{t x}+1\right)$ in single-user MIMO systems, where $C^{N_{t x}} N_{r x} N_{t x}$ is for matrix multiplication and $C^{N_{t x}} N_{r x}$ is for square operation $\left(N_{t x}\right.$ is antenna number in the transmitter, $N_{r x}$ is the antenna number of receiver). In the proposed scheme, we have to know the effective channel matrix which is a $n$ by $n \operatorname{matrix}\left(N_{t x}=\right.$ $N_{r x}=n$ ), so the complexity of ML detection in the proposed scheme is $C^{n} n(n+1)$ and the complexity of the simplified ML detection method is only $16 C, C$ is the constellation size.

We choose $C=4$ because of QPSK modulation in our proposed system. We can find out each mobile's complexity order in original ML is about 96 but just 64 of the proposed method.

\section{System Simulation Results}

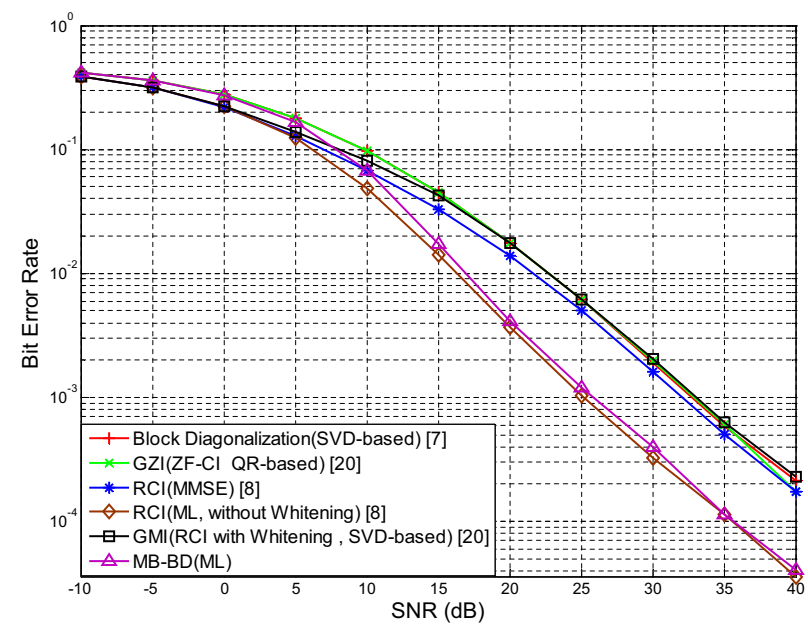

Fig. 3. BER comparison of proposed and other methods.

Figure 3 shows that the error performance of the proposed scheme outperforms BD, GZI, RCI-MMSE and RCI with whitening. MB-BD-ML can get about $6 \mathrm{~dB}$ gain as we set the constraint of BER rate at $10^{-3}$. We can see that the

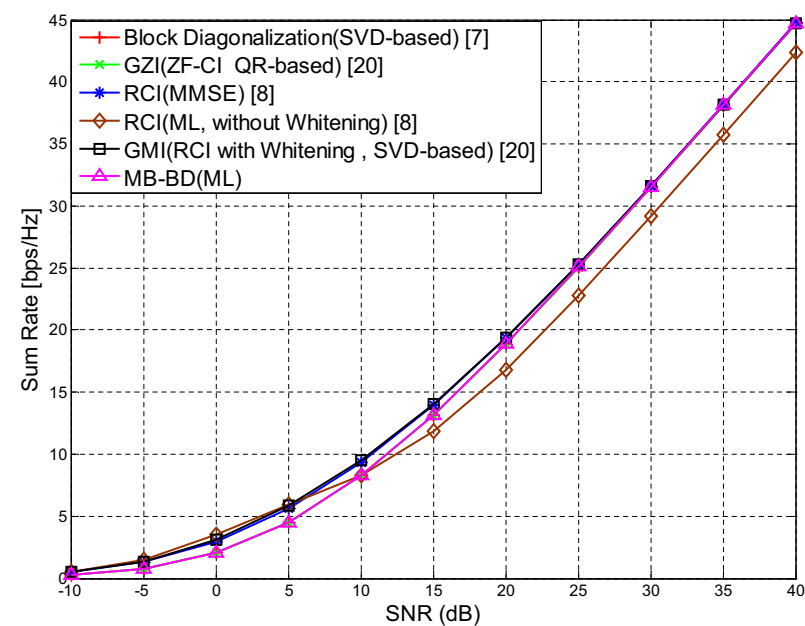

Fig. 4. Sum rate comparison of proposed and other methods.

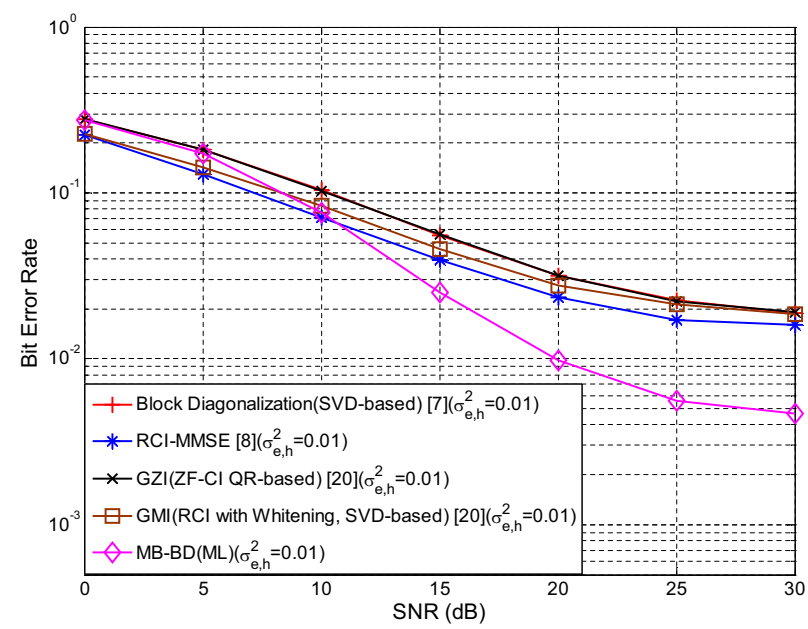

Fig. 5. BER comparison when channel state information with mean square error $(\mathrm{MSE})=0.01$ at transmitter.

BER performance of the proposed scheme is almost as the same as the RCI-ML. RCI [8] is based on MMSE criterion to maximize SINR of receiver but transmitter needs to know the noise power, so it has to do SNR estimation in practice. The performance will get worse if noise power estimation error occurs in SNR detector. The proposed method doesn't need to know the noise power, so it doesn't have the problem.

In Figure 4, GZI [20] achieves the performance as the same as conventional BD but with reduced complexity. GMI [20] uses noise-whitening to minimize the interference plus noise power, RCI-MMSE based on MMSE criterion to find a decoder at each receiver, so GMI and RCI-MMSE have higher sum rate than MB-BD, BD, and GZI. We can see that the sum rate of the proposed method approach to GMI and RCI-MMSE as SNR increases because the multiuser interference dominant the performance as SNR increases. In lower SNR, the sum rate of the proposed method (MB-BD) is lower than GMI and RCIMMSE because our method doesn't minimize the interference plus noise power. Comparing to RCI-ML (without whitening), 
the proposed method has better sum rate when SNR is larger than $10 \mathrm{~dB}$ but has worse performance when SNR is lower than $10 \mathrm{~dB}$. Figure 5 shows the proposed scheme (MB-BD) has more than $10 \mathrm{~dB}$ gain when we set constraint of BER at $10^{-2}$ as the mean square error (MSE) of channel gain is 0.01 .

\section{CONCLUSION}

The proposed multiuser spatial multiplexing scheme uses precoding to support multiuser in multiantenna wireless channels. The proposed novel maximum beamforming for block diagonalization (MB-BD) is designed for multiuser spatial multiplexing system, which uses sum space of the eigenvectors correspond to the first and second largest eigenvalues of channel matrix and combines QR decomposition for the zero interuser interference. BD is inferior in terms of the sum capacity to dirty paper coding (DPC), the simulation results show that the sum rate of proposed MB-BD scheme is similar to conventional $\mathrm{BD}$, but has better error performance than conventional BD. Comparing to BD, we can get about $37.5 \%$ reduction of computational complexity for computing the precoding matrix of each user; Comparing to original ML, we can get about $33.33 \%$ reduction of computational complexity at receiver. Comparing to other schemes, simulation results show that the proposed scheme is a good choice if we want to have better sum rate and error performance when SNR is larger than $10 \mathrm{~dB}$.

\section{REFERENCES}

[1] Q. H. Spencer, C. B. Peel, A. L. Swindlehurst, and M. Haardt, "An introduction to the multi-user MIMO downlink," IEEE Commun. Mag., pp. 60-67, Oct. 2004

[2] M. Costa, "Writing on dirty paper," IEEE Trans. Inf. Theory, vol. 29, no. 3, pp. 439 - 441, May 1983

[3] G. Caire and S. S. (Shitz), "On the achievable throughput of a multiantenna Gaussian broadcast channel," IEEE Trans. Inf. Theory, vol. 43, no. 7, pp. 1697-1706, Jul. 2003.

[4] H. Weingarten, Y. Steinberg, and S. S. (Shitz), "The capacity region of the Gaussian MIMO broadcast channel," IEEE Trans. Inf. Theory, vol. 52, no. 9, pp. 3936-3964, Sep. 2006.

[5] D. Gesbert, M. Kountouris, R. W. H. Jr., C.-B. Chae, and T. Saltzer, "Shifting MIMO paradigm: from single user to multiuser communications," IEEE Trans. Inf. Theory, vol. 24, no. 5, pp. 36-46, May 2007.

[6] C. B. Peel, B. M. Hochwald, and A. L. Swindlehurst, "A vectorperturbation technique for near-capacity multiantenna multiuser communication - part I: Channel inversion and regularization," IEEE Trans. Commun., vol. 53, pp. 195-202, Jan. 2005.

[7] Q. H. Spencer, A. L. Swindlehurst, and M. Haardt, "Zero-forcing methods for downlink spatial multiplexing in multiuser MIMO channels," IEEE Trans. Signal Process., vol. 52, no. 2, pp. 461-471, Feb. 2004.

[8] H. Lee, K. Lee, B. M. Hochwald, and I. Lee, "Regularized channel inversion for multiple-antenna users in multiuser MIMO downlink," in Proc. IEEE ICC '08, vol. 5, Beijing, China, May 2008, pp. 3501-3505.

[9] Z. Pan, K.-K. Wong, and T.-S. Ng, "Generalized multiuser orthogonal space-division multiplexing," IEEE Trans. Wireless Commun., vol. 6, pp. 1969-1973, Nov. 2004.

[10] S. Z. J. Zhang, Y. Wu and J. Wang, "Joint linear transmitter and receiver design for the downlink of multiuser MIMO systems," IEEE Commun. Lett., vol. 9, pp. 991-993, Nov. 2005.

[11] L. U. Choi, "Multiuser MISO and MIMO trasmit signal processing for wireless communication," PHD dissertation, University of Hong Kong, 2003.

[12] C. Tse, K.-W. Yip, and T.-S. Ng, "Performance tradeoffs between maximum ratio transmission and switched-transmit diversity," in Proc. 11th IEEE Int. Symp. Pers., Indoor Mobile Radio Commun. (PIMRC'2000), London, UK, Sep. 2000, pp. 1485-1489.
[13] H. Nishimoto, S. Kato, Y. Ogawa, T. Ohgane, and T. Nishimura, "Imperfect block diagonalization for multiuser MIMO downlink," in Proc. 19th IEEE Int. Symp. Pers., Indoor Mobile Radio Commun. (PIMRC'2008), Cannes, France, Sep. 2008, pp. 1-5.

[14] K. S. Gomadam, H. Papadopoulos, and C.-E. Sundberg, "Techniques for multi-user MIMO with two-way training," in Proc. IEEE ICC '08, vol. 5, Beijing, China, May 2008, pp. 3360-3366.

[15] R. A. Horn and C. Johnson, Matrix Analysis. Cambridge, U.K.: Cambridge Univ. Press, 1985.

[16] B. Hassibi, "An efficient square-root algorithm for BLAST," in Proc. IEEE Int. Conf. Acoustics, Speech, Signal Processing (ICASSP), vol. 2, pp. 5-9, Jun. 2000.

[17] G. H. Golub and C. F. VanLoan, Matrix Computations. Baltimore, MD: Johns Hopkins Univ. Press, 1989, 2nd ed.

[18] C. J. Huang, C. W. Yu, and H. P. Ma, "A power-efficient configurable low-complexity MIMO detector," IEEE Trans. Circuits Syst. I, vol. 56, no. 2, pp. 485-496, Feb. 2009.

[19] C. W. Yu and H. P. Ma, "A low complexity scalable MIMO detector," in Proc. IEEE IWCMC '06, Vancouver, British Columbia, Canada, July 2006, pp. 605-610.

[20] H. Sung, S.-R. Lee, and I. Lee, "Generalization of channel inversion algorithms for multiuser MIMO downlink systems," in Proc. IEEE ICC '08, vol. 5, Beijing, China, May 2008, pp. 3350-3354. 